\title{
A Power-Aware Routing Protocol Using Multi-Route Transmission for Mobile Ad Hoc Networks
}

\author{
Kuang-Han Fei, Sheng-Yan Chuang, and Sheng-De Wang \\ National Taiwan University \\ Department of Electrical Engineering \\ Taipei, Taiwan \\ sdwang@ntu.edu.tw
}

\begin{abstract}
We presents a power-aware routing protocol called MultiRoute Transmission Routing that utilizes multiple routes to transmit the data traffic simultaneously and leads to a balanced energy consumption. The proposed routing approach can extend the system lifetime of the network. A new routing decision index is also proposed for the route selection mechanism, which takes both the shortest-path and the maximum system lifetime into consideration and dynamically adjusts its weight between them according to the energy usage of the network. Experiment results show that, the proposed routing protocol provides a higher performance than other well-known power-aware routing protocols in terms of the energy-efficiency.
\end{abstract}

\section{Introduction}

Much research has addressed the energy-efficient issue of MANET routing protocols. We can roughly classify these solutions of the energy-efficiency protocol design into three categories: 1) Node energy state management: the mobile hosts can support a sleep mode that operates in a much lower energy level to conserve its battery energy. To maintain the traffic connectivity, these approaches usually keep few of hosts to active in order to buffer data packets for their sleeping neighbors. The approaches of SPAN [1] and GAF 2 belongs to this category; 2) Transmission power control: in the wireless transmission, the radio transmission power required is proportional to $d^{\alpha}$, where $d$ is the distance between the two hosts, and $\alpha$ is typically between 2 and 4 . In other words, the power consumption of transmission is at least the second order of the transmission range. The protocols in this approach try to achieve the goal of the minimum energy cost per packet transmission. Minimum Total Transmission Power Routing (MTPR) [3. prefers using routes with more hops and gets a shorter transmission range as compared to the traditional shortest-path manner. Other works about the approach can be found in [4, 5], 6] and 7]. The purpose of the approach is to minimize the power consumption during the packet transmission. However, it does not consider the residual energy usage of hosts in the network and cannot 
guarantee to extend the system lifetime of the network; 3) Power-aware routing: unlike the traditional shortest-path routing protocols, power-aware routing protocols realize the energy information of hosts. According to the energy information of hosts, power-aware routing protocols can take different power cost metrics for route selection into the consideration. The goal of this approach is to maximize the network lifetime by balancing the energy usage of the hosts. Some protocols consider the remaining energy of hosts as the metric in order to prolong the lifetime of the hosts, like Min-Max Battery Cost Routing (MMBCR) [8]. The MMBCR assumes that the cost of a route is the inverse of energy value which belongs to a host that has least remaining battery energy in this route. The route selection policy of the MMBCR is to choose a route which has the smallest route cost. Thus, the MMBCR may prefer a longer path that has the largest remaining battery energy to the shortest-path. But in this design of metric, it might consume more energy because the total transmission energy consumption of the longer path may be larger than that of the shortest-path. However, the MMBCR successfully extends the network lifetime due to a more balanced usage of the energy in the network. Conditional Max-Min Battery Capacity Routing (CMMBCR) 9] tries to consider both the minimum transmission energy cost and the maximum network lifetime in the route selection. The CMMBCR presents a hybrid method that selects a route favored by either the MTPR or the MMBCR by using a given threshold $\gamma$, which is a percentage value of hosts' initial energy between 0 and 100. When all hosts in possible routes have sufficient remaining battery energy (above the threshold $\gamma \times$ node's_initial_energy), a route with the minimum transmission energy cost is chosen. However, if all possible routes have low remaining battery energy (below the threshold), a route with the maximum remaining battery energy is chosen in order to prolong the hosts lifetime. Many of research issues are also addressed in this approach in recent years, such as [10], 11], 12] and [13].

In wired networks, multiple-path routing has been discovered with a lot of benefits, such as more balance traffic loading, more network end-to-end bandwidth etc. However, the advantages of multiple-path routing are not obvious in MANETs because the communications along multiple, different paths may interfere with each other due to the single-channel broadcast feature of the wireless radio transmission (i.e. not contention free). Using the multiple-path in DSR can maintain correct end-to-end transmission for a long time than a single path which has been proven in [14]. In [15], a diversity injection method has been proposed to find out more disjoint paths. This study also shows that multiple-path can balance network loads. In [16], the authors present a deep performance study of the multiple-path routing in MANETs. They also have proposed a new method to find disjoint paths to improve the performance of the multiple-path mechanism. In MANETs, the DSR and the Temporally Ordered Routing Algorithm (TORA) [17] store multiple paths for a particular destination as backup routing paths. However, we thought that using multiple paths as the backup routing paths is a kind of waste if the disjoint paths with sufficient remaining energy do exist. The proposed power-aware routing protocol, the PMRTR, efficiently 
utilizes these paths to simultaneously transmit the data packets, with a more balancing energy usage among nodes; thus, it extends the system lifetime of the network. To the best of our knowledge, we are the first to apply the concept of the multiple-path transmission to a power-aware routing protocol.

\section{Power-Aware Multi-Route Transmission Routing Protocol}

\subsection{Concepts of PMRTR}

Many power-aware routing protocols are based on the DSR protocol because of some characteristics of the DSR. First, the source node in the DSR has full knowledge about all possible route information in the network. Second, the mechanism of the DSR route discovery allows each intermediate node to attach their energy information (and network address also) in the RREQ and the RREP packets which can be piggybacked back to the source. These characteristics make it possible for the source node in the DSR to make a power-aware route selection according to the energy information extracted from the received RREP packets. The proposed power-aware routing protocol, the PMRTR, is also an enhancement over the DSR. The design goal of the PMRTR is to maximize the overall system lifetime in the network. To achieve that, we propose the following major improvements of the PMRTR over the DSR:

1) Energy information awareness: Each node should attach its current status of the energy into the RREQ and RREP packets during the route discovery stage. When a source receives the RREPs replied by a destination, it can extract the energy information of the route out and analyze the route cost later.

2) Energy-efficient route cost metric: The route selection not only considers the routing path length, but also the energy balance. The route cost metric in the PMRTR takes both the minimum transmission energy cost and the maximum network lifetime into consideration.

3) Multiple-path transmission: Unlike the DSR or any other power-aware routing protocols that also based on the DSR, the PMRTR uses multiple routes to simultaneously transmit the data packets to the destination. The packets that used to be transmitted through the single-path, now disperse to transmit along several different paths which all have approximate large residual energy capacity. Thus, since the traffic loading and energy depletion can be distributed, none of routes will be overused in the PMRTR.

4) Dynamic cost metric adjustment and route selection criterion: We adjust route cost metric by considering the balance between the minimum transmission power cost and the maximum network system lifetime according to the residual energy capacity of nodes. The route selection strategy uses a threshold and the degree of dissimilarity of routes to filter the routes with high cost out of candidate paths of the multi-route transmission. 


\section{$2.2 \quad$ PMRTR Protocol}

The residual energy capacity of a route in the PMRTR is defined as following. If we consider a generic route $r_{d}=\left\{n_{s}, n_{1}, n_{2}, \ldots, n_{d}\right\}$, where $n_{s}$ is the source node and $n_{d}$ is the destination node. Let $E_{i}(t)$ be the residual energy capacity of node $n_{i}$ at time $t$. We define the residual energy capacity of a route $r_{j}$ : $R E\left(r_{j}\right)=\min _{\forall n_{i} \in r_{j}} E_{i}(t)$, in other words, the least energy of the node on this route. Although the design goal of the routing cost metric in the PMRTR is to try to take both the minimum transmission power cost and the maximum system lifetime of the network into consideration. However, the experiment results in analysis of power-aware routing protocols 18 shows that the shortest-path routing protocol (i.e. in the DSR) surprisingly performs great in energy-efficiency. This interesting result shows that even the shortest-path routing is not designed for energy-efficiency, it still may outperform than other power-aware routing protocols in some scenarios. The reasons of the energy efficiency of the shortestpath are 1) the energy consumption of the shortest-path approximates with the minimum transmission power cost in the case that each node in the network operates with same transmission power, and 2) the high mobility of the ad hoc networks makes the traffic loading natural evenly distributed among the nodes in the network. In addition, we should also note that since only a few actual network interface cards allow several discrete power levels, to assume that transmission range/power can be continuously configured is not practical in the real wireless network environments. To be specific, the PMRTR route cost metric is determined by both the hop counts of the route and the residual energy capacity of the route. Consider two paths $r_{1}$ and $r_{2}$ in Figure 1. There are two paths

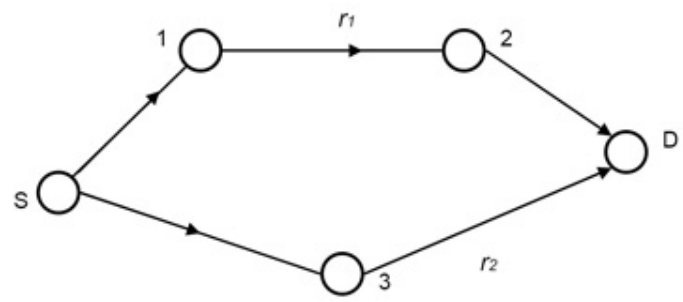

Fig. 1. A simple network topology for the illustration of routing cost metric

$r_{1}=\left\{n_{s}, n_{1}, n_{2}, n_{d}\right\}$ and $r_{2}=\left\{n_{s}, n_{3}, n_{d}\right\}$ in the network, where $n_{s}$ is the source and $n_{d}$ is the destination. Assume that the energy consumption of any link is $P$, $m_{1}$ is the hop counts of $r_{1}$, and $e_{1}$ is the residual route energy of $r_{1} ; m_{2}$ is the hop counts of $r_{2}$, and $e_{2}$ stands for the residual route energy of $r_{2}$. The updated residual energy of the route $r_{1}$ is $e_{1}^{\prime}=e_{1}-P m_{1}$ if the route $r_{1}$ is taken and the updated residual energy of the route $r_{2}$ is $e_{2}^{\prime}=e_{2}-P m_{2}$.

As we can see, the factor $P$ plays an important role in determining the contribution proportion between the hop counts and the residual energy of the route in the route selection. However, if we define the factor $P$ as the energy consumption of the host-to-host transmission power in $\mathrm{mW}$, the route selection will 
prefer much the shortest-path to the paths with large residual energy because the typical value of the energy consumption of the host-to-host transmission power is constant while the residual energy is decaying. Therefore, we can add a weighting $K$ to the the factor $P$ such that the balance can be achieved between the hop counts and the route residual energy by setting the weighting $K$ as a variable with its value proportional to the residual energy of the nodes in the network. In this way, we can define a decision index $I_{i}$ to evaluate the priority of route $r_{i}$ as follows:

$$
I_{i}=e_{i}-K P m_{i}
$$

where $K$ is a weighing to be adaptively determined in the networking environment. It is obviously that we prefer a route with largest $I_{i}$ among all available routes. Now, it remained to determine the weighting $K$. Since the residual energy of nodes decreases as time goes by, it is natural to set the weighting $K$ be related to the residual energy of nodes. To this end, we consider a threshold energy $e_{t h}=T H_{C M} \times E_{\text {init }}$, where $T H_{C M}$ is a constant and set weighing $K$ as follows:

$$
K= \begin{cases}K_{\text {init }} & \text { if } e_{i}>e_{t h} \\ K_{\text {init }} \times \frac{e_{i}}{e_{t h}} & \text { if } e_{i}<e_{t h}\end{cases}
$$

where $K_{\text {init }}$ is the initial value. Thus, when the residual energy capacity of nodes in the network is critical, the route selecting policy of the PMRTR concern about the residual energy capacity than the hop counts of the routes.

The multiple-route transmission uses different paths to transmit simultaneously, dispersing the traffic load into these paths. In traditional multiple-route routing protocols, the paths being used for multiple-route transmission should be the disjoint paths. The numbers of disjoint paths strongly affect the performance of the multiple routes transmission. However, the availability of the paths that are entirely disjointed may be rare in some network scenarios; hence the improvement of the multiple routes transmission is not significant. In the PMRTR, we allow multiple routes transmission upon the paths which are not necessary to be completely disjointed. We propose a new criterion, the routes dissimilarity, which indicates the differentiation between the different paths. The routes dissimilarity between two routes $\left(r_{a}\right.$ and $\left.r_{b}\right)$ is defined as:

$$
R D\left(r_{a}, r_{b}\right)=\frac{M L\left(r_{a}, r_{b}\right)-S N\left(r_{a}, r_{b}\right)}{M L\left(r_{a}, r_{b}\right)},
$$

where the $M L\left(r_{a}, r_{b}\right)$ denotes the length of the shorter route and the $S N\left(r_{a}, r_{b}\right)$ denotes the number of same nodes between the $r_{a}$ and the $r_{b}$.

For example, suppose we have a set of routes $R=\left\{r_{1}, r_{2}, r_{3}, r_{4}\right\}$, where $r_{1}=\left\{n_{s}, n_{1}, n_{d}\right\}, r_{2}=\left\{n_{s}, n_{2}, n_{3}, n_{4}, n_{5}, n_{d}\right\}, r_{3}=\left\{n_{s}, n_{2}, n_{7}, n_{8}, n_{9}, n_{d}\right\}$ and $r_{4}=\left\{n_{s}, n_{4}, n_{d}\right\}$. The routes dissimilarity between $\left\{r_{1}, r_{2}\right\},\left\{r_{2}, r_{3}\right\}$ and $\left\{r_{1}, r_{4}\right\}$ are $R D\left(r_{1}, r_{2}\right)=\frac{(3)-(2)}{(3)} \cong 0.33, R D\left(r_{2}, r_{3}\right)=\frac{(6)-(3)}{(6)}=0.5$ and $R D\left(r_{1}, r_{4}\right)=$ $\frac{(3)-(2)}{(3)} \cong 0.33$ respectively. Let $r_{5}$ be a route that we just discovered and the 
route set $R$ is the routes stored in the MRT for certain destination. The average routes dissimilarity between $r_{5}$ and $R$ is:

$$
R D\left(r_{5}, R\right)=\frac{\sum_{\forall r_{i} \in R} R D\left(r_{5}, r_{i}\right)}{\bar{R}}
$$

where $\bar{R}$ is the number of routes in the route set $R$. Using with routes dissimilarity instead of the traditional disjoint definition as the criterion of selecting available paths, the performance improvement in multiple routes transmission can be seen more significantly in different network scenarios.

\section{Experiment Result}

Since the main goal of the proposed PMRTR is to balance the energy consumption of the nodes in a network and thus to maximize system lifetime of the network, we consider two essential criteria: 1) System lifetime of the network, which is commonly defined as the time of the first node that runs out off its energy in the network; and 2) Standard deviation of the nodes' energy in the network, which reflects the distribution of the residual energy of nodes in the network.

\subsection{Simulation Model}

We use the ns-2 [19] which is a discrete event driven simulator developed by University of California at Berkeley and VINT project as our simulation platform. We also use the wireless extensions provided by CMU [20]. The setup consists of a test bed of 24 nodes in a $600 \times 600 \mathrm{~m}^{2}$ area. The communication range of each node is assumed to be $250 \mathrm{~m}$. We separate these 24 nodes into 20 intermediate nodes and 4 traffic nodes. These 4 traffic nodes which have fixed locations in the four corners of the simulation area and act as the sources and the sinks (destinations) that continuously exchange the data packets to each other. The 20 intermediate nodes which are confined in simulation environment perform serve as the forwarding nodes in the ad hoc mode.

The traffic nodes in diagonal communicate with each other by establishing a $4 \mathrm{pkt} / \mathrm{sec}$ CBR traffic connection with $512 \mathrm{~KB}$ packet size at simulation beginning. In other words, every intermediate node in the network is covered by four CBR traffic communications all the time. The design propose of this simulation scenario is to isolate the source/destination nodes from the intermediate nodes. In this isolation process, if a node runs out off its energy, it must do because this node is responsible for some traffic forwarding which is caused by some bad route selection of power-aware routing protocols, and not because that node was the source or the destination which belongs to some traffic. By observing the energy usage of every intermediate node, we can accurately measure the energy-efficiency performance of the power-aware routing protocols easily.

For every intermediate node, three kinds of mobility, low (in $1 \mathrm{~m} / \mathrm{s}$ ), medium (in $5 \mathrm{~m} / \mathrm{s}$ ) and high (in $10 \mathrm{~m} / \mathrm{s}$ ), are investigated. In each mobility pattern, the random waypoint model with constant pause-time 30 seconds is applied. 
It means that after every node reaches the current waypoint, it pauses for 30 seconds, and then randomly chooses its next waypoint. The standard deviation of nodes' energy is measured in every 30 seconds. We average these results before the network lifetime and report this value as the energy standard deviation of the protocol.

We should notice the significant energy consumption of the overhearing between nodes in mobile ad hoc networks through the experiment results in [21. Due to the nonexistence of any wireless base station or central controller which can be in charge of buffing the traffic packets for sleeping neighbors in current power-aware routing protocols, the nodes cannot sleep in the ad hoc mode. Because of that, all of the mobile nodes will consume energy unnecessarily due to overhearing the transmission of their neighbors. Although the overhearing feature obviously wastes lots of valuable energy resources of nodes, the nodes of power-aware routing protocols based on on-demand manner should remain active all the times so as to participate in the route discovery by broadcasting the RREQ and the RREP packets. Somehow, under some dense network environments, the energy consumption of the overhearing engages around $80 \%$ of total energy consumption of the whole network [21. However, we do not disable the overhearing feature in any of our experiments due to more practical reflecting the actual network environment behaviors.

The parameters in the PMRTR protocol are assigned as following setup: the weighting factor $K_{\text {init }}$ of the routing decision index $I$ is $10 \%$, and the threshold $T H_{C M}$ in the weighting factor $K$ is set as $25 \%$ of the initial energy of nodes (i.e., $T H_{C M}=25 \%$ ). In our experiments, all intermediate nodes have their initial energy which are randomly selected in $200 \pm 0.5$. Each experiment result is obtained from 20 different runs at least. The simulation time in each run is set to 1000 seconds.

\subsection{Power-Aware Routing Protocols Comparison}

In the experiment of this sub-section, we compare the energy-efficiency performance with the shortest-path routing, the PMRTR, and two well-known poweraware routing protocols, such as the MMBCR and the CMMBCR. We fully implement the functionalities of the MMBCR and the CMMBCR (with that threshold is half of the initial energy of nodes) in the ns- 2 and also modify the route maintenance procedure of these protocols. The mechanism that protocol will flush its route cache and establish a new route discovery for any active transmission in order to update the route information and the energy usage information is added into the route maintenance procedure of these routing protocols. Since the PMRTR is also a routing protocol, the criteria of traditional routing protocols such as the delivery ratio and the end-to-end delay should also perform with maintaining in a good condition. Besides the system lifetime of network and the average energy standard deviation, we also measure and compare the delivery ratio and the end-to-end delay of these protocols. The system lifetime comparisons of the candidate protocols are illustrated in Figure 2, while the average energy standard deviation comparisons are shown in 
System Lifetime of Network in Protocols Comparison (Seconds)

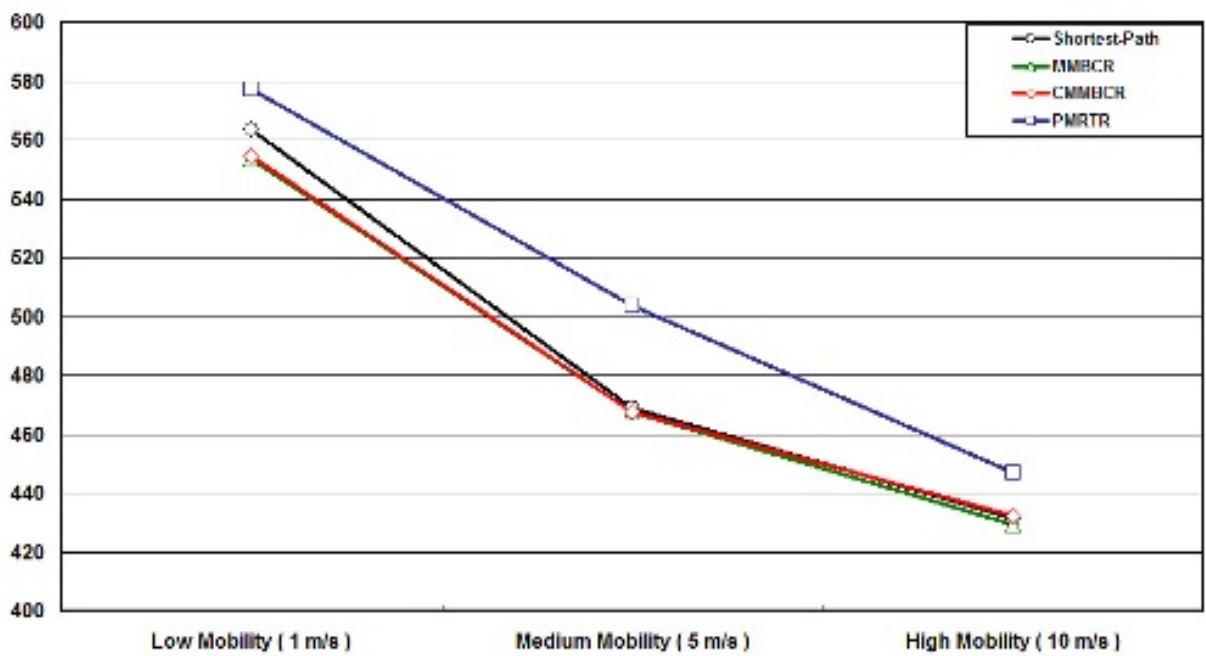

Fig. 2. System Lifetime for Protocols Comparison

Average Energy Standard Deviation of Network in Protocols Comparison (Joules)

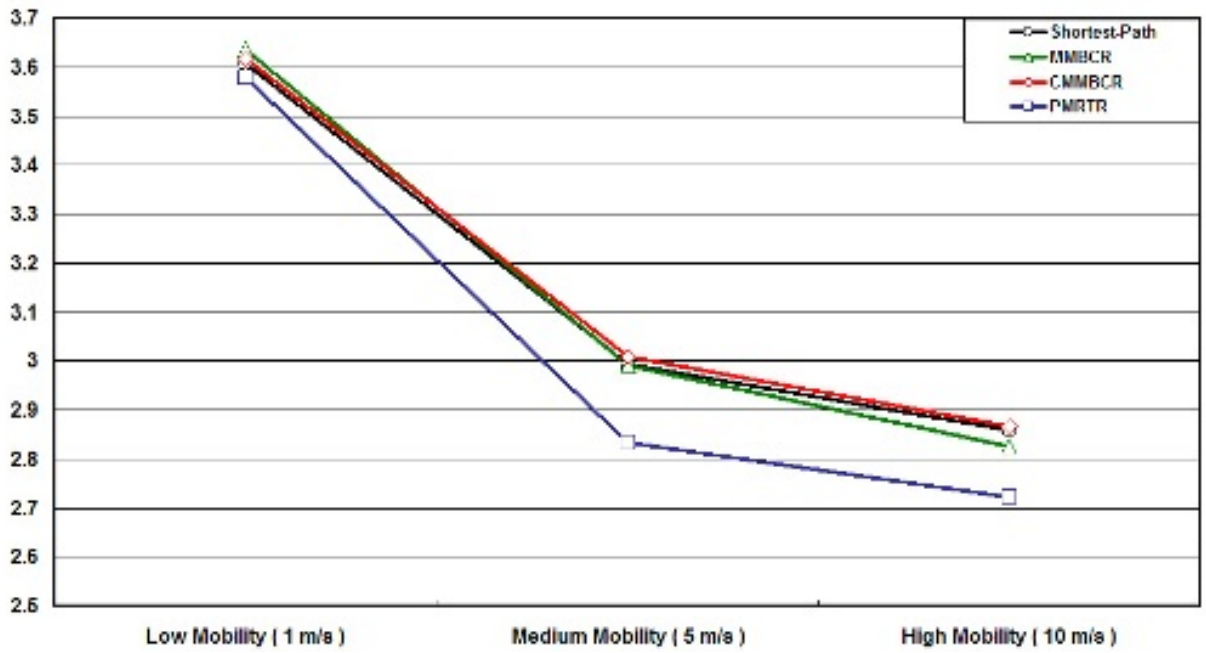

Fig. 3. Average Energy Standard Deviation for Protocols Comparison

Figure 3. The simulation results show that, the PMRTR performs the longest system lifetime in every mobility scenarios. It proves that the concept of the multi-route transmission and our novel routing cost metric lead to more balanced energy consumption, thus, obviously extends the system lifetime of the network. However, the difference between the simulation results of protocols is 
not quite large because of the significant influence of the overhearing power consumption.

In regard to the average energy standard deviation, the PMRTR also shows the best results, which provide most evenly energy usage between nodes in the network. Through this simulation, we can see that the PMRTR is the most fairness protocol in balancing the energy consumption among these candidate power-aware routing protocols. We also note that, as can be seen in the Figure 3 , the average energy standard deviation decreases when the mobility of the network increases because that the energy consumption which is caused by the traffic can be more evenly distributed to different nodes through the high network mobility.

\section{Conclusion}

In this paper, the Power-aware Multi-Route Transmission Routing (PMRTR) protocol for MANETs is proposed. The PMRTR uses the concept of the multiplepath transmission to evenly distribute the traffic loading among the mobile nodes in the network, thus maximizing the system lifetime of the network. The route selection mechanism is based on a routing cost metric that takes both the shortest-path and the maximum system lifetime into consideration and dynamically adjusts the weightings between them according to the energy usage status of the network. The experiment results show that, the PMRTR provides longer system lifetime and more balanced energy consumption between the mobile nodes than the shortest-path routing and other power-aware routing protocols. In addition, the proposed approach can also keep a good delivery ratio and a low end-to-end delay.

\section{Acknowledgments}

The work was partially supported by MediaTek Inc. Taiwan and partially supported by National Science Council, Taiwan, under the grant no. NSC 94-2213E-002-003.

\section{References}

1. Chen, B., Jamieson, K., Balakrishnam, H., Morris, R.: Span: Energy-efficient coordination for topology maintenance in ad hoc networks. ACM Wireless Networks Journal 8 (2002)

2. Xu, Y., Heidemann, J., Estrin, D.: Geography-informed energy conservation for ad hoc routing. Proceedings of the Seventh Annual ACM/IEEE International Conference on Mobile Computing and Networking (2001)

3. K.Scott, N.Bambos: Routing and channel assignment for low power transmission in PCS. ICUPC '96 (1996)

4. Doshi, S., Bhandare, S., Brown, T.X.: An on-demand minimum energy routing protocol for a wireless ad hoc network. ACM SIGMOBILE Mobile Computing and Communications Review (2002) 
5. Stojmenovic, I., Lin, X.: Power-aware localized routing in wireless networks. IEEE Transactions on Parallel and Distributed Systems (2001)

6. Zhang, B. Mouftah, H.T.: Localized power-aware routing for wireless ad hoc networks. IEEE International Conference on Communications (2004)

7. Li, J., Cordes, D.: Hybrid greedy-multicasting power-aware routing protocol in ad hoc networks. International Conference on Information Technology: Coding and Computing (ITCC'04) 2 (2004)

8. Singh, S., M.Woo, Raghavendra, C.S.: Power-aware routing in mobile ad hoc networks. Proceedings of Mobicom (1998)

9. Toh, C.K.: Maximum battery life routing to support ubiquitous mobile computing in wireless ad hoc networks. IEEE Communications Magazine (2001)

10. Maleki, M., Dantu, K., Pedram, M.: Power-aware source routing protocol for mobile ad hoc networks. International Symposium on Low Power Electronics and Design, and Proceedings of the 2002 international symposium on Low power electronics and design (2002)

11. Sheu, J.P., Lai, C.W., Chao, C.M.: Power-aware routing for energy conserving and balance in ad hoc networks. Proceedings of the 2004 IEEE International Conference on Networking, Sensing \& Control (2004)

12. Zhou, Y., Laurenson, D.I., McLaughlin, S.: High survival probability routing in power-aware mobile ad hoc networks. IEE Letters 40 (2004)

13. Wang, K., long Xu, Y., liang Chen, G., feng Wu, Y.: Power-aware on-demand routing protocol for MANET. 24th International Conference on Distributed Computing Systems Workshops (2004)

14. Nasipuri, Das, S.: On-demand multipath routing for mobile ad hoc networks. Proceedings of the 8th int. Conf. On Computer Communications and Networks(IC3N) (1999)

15. Pearlman, M., Z.J. Hass, P.S., Tabrizi, S.: On the impact of alternate path routing for load balancing in mobile ad hoc networks. Proceedings of IEEE/ACMMobiHoc (2000)

16. Wu, K., Harms, J.: Performance study of a multi-path routing method for wireless mobile ad hoc networks. Proceedings of the Ninth International Symposium in Modeling, Analysis and Simulation of Computer and Telecommunication Systems (MASCOTS'01) (2001)

17. Park, V.D., Corson, M.S.: A highly adaptive distributed routing algorithm for mobile wireless networks (TORA). IEEE Infocom (1997)

18. Safwat, A., Hassanein, H., Mouftah, H.: Energy-aware routing in MANETs: Analysis and enhancements. ACM Workshop on Modeling, Analysis and simulation of Wireless and Mobile Systems (2002)

19. http://www.isi.edu: The ns-2 network simulator. (obtain via http://www.isi.edu/ nsnam/ns/)

20. CMU: Cmu monarch extensions to ns. (obtain via http://www.monarch.cs.cmu. $\mathrm{edu} /$ )

21. Cano, J.C., Kim, D.: Investigating performance of power-aware routing protocols for mobile ad hoc networks. International Mobility and Wireless Access Workshop (MobiWac'02) (2002) 